\title{
The Emergence of Paradigm Setters through Firms' Interaction and Network Formation
}

\author{
Rainer Andergassen ${ }^{1}$, Franco Nardini $^{2}$, Massimo Ricottillit ${ }^{1,3}$ * \\ ${ }^{1}$ Department of Economics, University of Bologna \\ P.zZa Scaravilli 2, 40126 Bologna, Italy \\ ${ }^{2}$ Department of Mathematics for the Social Sciences, \\ University of Bologna, \\ Viale Filopanti 5, 40126 Bologna, Italy \\ ${ }^{3}$ Centro Interdipartimentale Luigi Galvani, Bologna, Italy \\ ABSTRACT. Technological innovation requires the gathering of information through a \\ process of searching and learning. We distinguish two different but definitely complementary \\ and overlapping ways through which searching and learning occur. The first exploits the \\ spillover potential that lies in a firm's network and thanks to which gathering innovation- \\ useful information is actually possible. The second is the autonomous capacity that a firm \\ possesses in order to carry out in-house innovative search. We build a model where rationally \\ bounded firms try to increase their innovative capability through endogenous networking. \\ The paper characterizes the emergence of technological paradigm setters in terms of network \\ properties as they result from searching routines, furthermore the corresponding average \\ efficiency of the system in terms of innovative capability is assessed.
}

JEL classification numbers: L14, O33.

Keywords: Networks, Bounded Rationality, Technological Change, Innovative Capability, Paradigm Setters.

${ }^{*}$ Corresponding author. E-mail addresses: anderga@economia.unibo.it (R. Andergassen), nardini@dm.unibo.it (F. Nardini), ricottilli@economia.unibo.it (M. Ricottilli). 


\section{Introduction}

Technological innovation requires the gathering of information through a process of searching and learning. In economies at the cutting edge of their frontier the competitive drive compels leading firms to engage in this process lest their advantage be lost to competitors and imitators. This quest for information is largely an adaptive, gradual process in which internal, in-house resorces generating innovation-worthy knowledge are woven together with that obtained through technological spillovers proceeding from other firms. Firms, and agents within firms, exhibit bounded rationality and their success is largely due to but also constrained by their technological capabilities that are, nevertheless, magnified by interaction with other firms. The implication is that the process of search is local and confined within a neighbourhood that is cognitively reachable and that is, therefore, the medium of effective information flows. To this effect, the role of a network in which firms nest is paramount

Because of bounded rationality and radical uncertainty, the process of reciprocal observation and learning occurring through interaction between firms and making the spreading of technological spillovers possible takes place in.viable networks. Their importance has been highlighted in recent leterature investigating technological and knowledge diffusion, see for example, Cowan and Jonard (2004), Silverberg and Verspagen,(2002), Arenas et Alii,(2001, 2002). Networks, however, are subject to change and growth : connectivity between members, linkages between firms,can be viewed as a process in time. Seminal work in this field has been done by Albert and Barabasi, (2002)for an exaustive review, Watts and Strogatz (1998), for the emergence of small worlds. When seen as an interactive system (Kirman 1997a,1997b ), the economy generates capabilities that are mutually acquired rendering firms technologically interdependent. It must, nevertheless, be recognised that capability-building also depends on in-house efforts supported by investment in formal and informal research and development. In this paper we treat technological capabilities as a stock of firm-specific knowledge cumulated through time by every single 
firm in the economy. We, accordingly, propose a model in which it is posited that each firm is endowed with a measurable index of innovation-enabling knowledge that is subject to change as a consequence of an active searching policy. We distinguish two different but definitely complementary and overlapping ways through which searching and learning occur. The first exploits the spillover potential that lies in a firm's network and thanks to which gathering innovation-useful information is actually possible. The second is the autonomous capacity that a firm possesses in order to carry out in-house innovative search. While these two searching processes not only co-exist but are also reciprocally sustaining, we find it expedient to separate them by integrating a knowledge diffusion mechanism that propagates technological capabilities with an independent stochastic process capturing innovation arrivals due to internal R.\&D. A network's evolution depends on how firms assess its performance in terms of innovation-enabling spillovers. In a bounded rationality framework, firms normally explore a limited part of the firms' space and require a protocol to target their information gathering efforts. The paper addresses this issue by designing a routinised behaviour according to which firms periodically reshape the neighbourhood that they observe to glean information by reassessing other firms' contributions to their own capability. The way the specific neighbour-choosing routine is accordingly organised determines in a significant way firms' average innovative capability. This feature is modelled by changing the span of observation from a very broad setting, the whole economy, to a very narrow one, namely the most proximate neighbourhood membership. As a result of the structure of the model presented in the next section, there are two distinct but to some extent overlapping neighbourhoods which are relevant for firms' interaction. The first is the neighbourhood whose members are observed by each firm and from which capability contributions are obtained. We term this neighbourhood inward. The second is the one made up by firms observing and learning from information flowing from another neighbouring firm: it evolves as an active search for new inward members is carried out. We call this neighbourhood outward. This process of information interaction leads to the emergence of some firms that are mostly observed by others thus providing some or much of their 
technological capability. It is these firms that we term paradigm setters. We also assume that in-house acquired capability is subject to structural shifts by means of periodic random shocks.

To keep the model mathematically tractable, we formalise the features stated above by means of a linear system in which technological capabilities are made to depend on a matrix of interaction with evolving outward neighbours as well as on a vector of in-house generated knowledge. The model is then simulated to determine the emergent properties of neighbourhood formation and stability together with average capability. We aim to identify (i) under what conditions the emergence of technological paradigm setters occurs, (ii) the pattern of neighbourhood formation and (iv) the average relative efficiency in terms of technological capability of the economy as a whole.

The plan of the paper is as follows: section two illustrates the linear model that is implemented to run simulations; section three describes the simulation procedures and the indexes employed to assess results; section four discusses results obtained and section five draws conclusions and sets an agenda for further research.

\section{Firms' technological capabilities and spillover potential}

A firm's technological capability is the upshot of an evolutionary process owing to learning, searching and gathering of information. It is, indeed, these capabilities that ultimately lead to that to innovate, much being explained by interaction taking place within the system. These three categories, however, are largely overlapping since neither can exist without the two others. In this section, we direct our analysis to investigate firms that are technological leaders and whose major interest lies with innovation. We, accordingly, assume that they possess 'in house' innovative capabilities resulting from past investment and that we, therefore, distinguish in a somewhat artificial manner but useful for modelling, from those that are entirely due to spillover from other firms' own. These capabilities can be viewed and measured in a way akin to the more general category of 
a firm's knowledge base, cognitive potential or set of skills, know-how and competencies: they can actually be modelled as either a vector arranging different indicators or more simply as a scalar compounding the whole. We choose the latter approach.

Let $V_{i}(t)$ be the scalar that at time $t$ designates firm $i$ 's innovative capability or, to use a term borrowed from biology, its innovative fitness. Then, $V(t)$ is the vector $V(t)=\left[V_{i}(t)\right], i=1,2 \ldots J$ arraying the fitness of all firms in the economy. By $C_{i}(t)$ we further designate the in-house capability cumulated until time $t$. As mentioned above, the latter, while embodying cumulated knowledge, requires investment to be preserved and eventually improved. Considerable efforts are therefore necessary to remain on the forefront of technological prowess; efforts which need not always prove successful. It is, accordingly, assumed that $C_{i}(t)$ be stochastically subject to change and $C_{i}(t) \in(0,1) . C(t)$ is the corresponding vector.

A significant part of total technological capability is explained by firms' interaction with other firms. As mentioned above, this is due to searching activity and to the ease with which each firm is observable by other firms when broadcasting information of its own innovative capability. For searching to be significant and transmission possible, it is necessary that cognitive proximity generate interaction to let an effective spill over take place. The ensuing capacity to broadcast relevant technological information may be measured by a basic index specific to each pair $i j$ of firms in the economy. Accordingly, let $a_{i j}$ indicate this index in terms of the part of each firm $j$ 's total innovative fitness that can cognitively be passed on to firm $i$ should the latter be in a position to observe the former. The entire web of interfirm technological spill over capacity can then be designated by a square, $J x J$, matrix $A$. The main diagonal of this matrix is made up by 0's, $a_{i i}=0$, since no firm broadcasts information to itself. Matrix $A$ is simply an indicator of how well observing firms understand the technology of other firms and, therefore, it states no more than a spill over potential as a structural characteristic of the economy.

Bounded rationality restricts the number of neighgbours that a firm can usefully search to glean technological information and furthermore it hinders an optimal choice of a new nieghbour when 
a firm gets the chance to adjust its neighbourhood.. Actual observation is restricted to a more or less narrow neighbourhood made up by firms whose informative usefulness has been discovered by a searching process. We accordingly postulate that firms carry out an active search to single out neighbours best suited to pass on innovative capability. It is assumed that each firm $i$ searches among its potential information suppliers $j$ whose broadcasting capacity is $a_{i}=\left(a_{i j}\right), j=1,2 \ldots J$, those that at each point in time it is able to choose as a target for innovative information and actually observe. This choice can be formalised by introducing the proximity matrix $B(t)=\left[b_{i j}(t)\right]$ where each $b_{i j}(t)=1$ or $b_{i j}(t)=0$ according to whether neighbour $j$ has been or hasn't been identified as a useful contributor. This procedure defines matrix $M(t)=\left(a_{i j} b_{i j}(t)\right)$. Thus, the innovative capability that is determined by interaction can be formalised by the system $M(t) V(t)$ where actually observed firms are restricted to a limited number of neighbours. The general equation for firm $i$ 's innovative capability is ${ }^{1}$

$$
V_{i}(t)=\sum_{j=1}^{J} a_{i j} b_{i j}(t) V_{j}(t)+C_{i}(t)
$$

The system describing innovative capability in time is

$$
V(t)=[I-M(t)]^{-1} C(t)
$$

where $[I-M(t)]^{-1}$ plays the role of an endogenous matrix multiplier of in-house capabilities.

Since firms are bounded in their rationality, different neighbourhood relationships lead to a different multiplier. This change occurs because firms attempt to improve their capabilities through networking.

\subsection{Neighbourhood structure}

The structure in which we describe firms' innovative capability can be represented by a directed graph of $J$ nodes each of which is connected with other nodes in two different but overlapping ways. The first is the number of connections that each firm establishes when observing other firms

\footnotetext{
1 Absorbing the impact of spillovers is clearly a process that requires an adjustment in time. We simplify this problem by assuming that the time required to complete adjustment is negligible in relation to the system evolution
} 
to determine its own innovative capability. The number of $k_{i, \text { in }}<<J$ connections defines for firm $i$ the dimension of its inward neighbourhood. This number is substantially smaller than $J$ since searching is costly and observation bounded. This neighbourhood can formally be defined as

$$
\Gamma_{i}(t)=\left\{j: j=1,2 \ldots J \wedge b_{i j}(t)=1\right\}
$$

This is the set of firms from which at any time $t$ firm $i$ is able to glean innovative capability through observation and learning and it forms the neighbourhood resulting from their active searching as they pursue improvement.

The second kind of neighbourhood, which we term the outward neighbourhood, is made up for each firm $j$ by firms that actually observe it. It passively results as a consequence of their networking activity. Let this neighbourhood be defined by:

$$
\Psi_{j}(t)=\left\{i: i=1,2 \ldots J \wedge b_{i j}=1\right\}
$$

Its size determines the impact of an observed firm's technological capacity as it propagates throughout the economy contributing to overall performance. For this purpose, we classify the population of firms according to classes of their outward neighbourhood size and thus define an impact factor by ranking them in terms of the number of outward neighbours:

Definition 1 Technological paradigm setters emerge when the probability of each rank of the impact factor is positive.

\subsection{Evolution}

Given this neighbourhood structure, evolution owes to two basic determinants: search routines and exogenous changes on individual firms' in-house innovative capabilities. Firms construe their inward neighbourhood by an active search aimed to single out members that contribute capability to their own. This search, while bounded by the neighbourhood in which the firm happens to be nested, may take place according to a variety of algorithms. We have chosen one that responds to the criteria of bounded rationality and satisficing. We propose two versions that respectively capture a strong and a weak form of bounded rationality. In both, we first conjecture that the 
cardinality of $\Gamma_{i}$ is $\left|\Gamma_{i}\right|=k_{i, i n} \ll J$ and generate the choice of neighbours and the evolution of this neighbourhood according to the following routine: each firm $i$ assesses the fitness contribution of its existing neighbours and picks out the least contributing one:

$$
\gamma_{i}(t-1)=\arg \min _{j \in \Gamma_{i}(t)}\left[a_{i j} b_{i j}(t-1) V_{j}(t-1)\right]
$$

Secondly, the identified neighbour is substituted with a new one by randomly drawing among the limited number of the latter own neighbours, in the case of weak bounded rationality, or by randomly drawing among the remaining $J-k_{i, i n}-1$ members of the entire economy, in the case of strong but bounded rationality. In either case, to generate a new $\Gamma_{i}(t)$ it is necessary that this simple condition be satisfied:

$$
V_{i}(t)>V_{i}(t-1)
$$

This procedure redefines at each time step $M(t)$ and the system then generates a new set of solutions.

Next to the dynamics generated by neighbourhood adjustment we introduce in the system the autonomous and independent dynamics involving the in-house capability $C(t)$. This vector is subject to change by a random draw of some $i \in(1,2 \ldots J)$ and by randomly redefining the $i^{t h}$ component by a new random value $C_{i}(t)$ uniformly chosen between 0 and 1 . These occurrences are arrivals that take place according to a predetermined mean waiting time $\mu$.

\section{Parameters and benchmarking}

The purpose of this model is to study the emergent properties of neighbourhood formation their stability and impact on average technological capabilities. In order to check for the impact of randomness in firms' searching activity we simplify the model by assuming parameters that insure an even field of equal starting points. It is, accordingly, conjectured at first that all firms have the same skill in broadcasting information and in spilling over their innovative capability. Matrix $A$ will, therefore, be set at $A=\left(a_{i j}=a\right), \forall i, j$. Parameter $a$ measures the strength of interaction 
and the degree to which each firm's innovative capability depends on other firms', given a matrix $M(t)=\left[a b_{i j}(t)\right]$. For the system to yield positive solutions for any given vector $C(t)$ and prevent explosive behaviour in $V(t), a$ must be kept as $a \leq \frac{1}{k_{i, i n}}, \forall i$. We further assume that the number of inward neighbours is the same for all firms and constant in time: $k_{i, i n}=$ const, $\forall i$.

In order to make a significant assessment of innovative performances as simulations are carried out, it is expedient to set benchmarks against which they can be measured. We propose the following definitions.

Definition 2 Let the vector $\bar{C}(t)$ be the vector of elemnts of $C(t)$ rearranged in decreasing order and let the matrix $\bar{B}=\left[b_{i j}\right]$ where $b_{i j}=1, \forall i=1,2 \ldots k_{i n}, \forall i \neq j, j \leq k_{i n}+1$ and $b_{i j}=1, \forall k_{i n}+$ $1 \ldots . . . J ; b_{i j}=0$ otherwise. Furthermore define $\bar{v}(t)=\frac{1}{J} \sum_{j=1}^{J} \bar{V}_{j}(t)$, where $\bar{V}(t)=[I-\bar{M}]^{-1} \bar{C}(t)$ and $\bar{M}=a \bar{B}$.

Definition 3 Let matrix $\underline{B}=\left\{b_{i j}=\frac{k_{i n n}}{J-1}, \forall i \neq j, b_{i i}=0 \forall i\right\}$. We then define $\underline{v}(t)=\frac{1}{J} \sum_{j=1}^{J} \underline{V}_{j}(t)$ ,$\underline{M}=a \underline{B}$ and $\underline{V}(t)=[I-\underline{M}]^{-1} C(t)$.

Remark 4 If $\bar{V}(t)$ can be considered as the capability values resulting from an optimally planned network, $\underline{V}(t)$ is the average outcome from a random choice of neighbours.

Definition 5 Given that $v(t)=\frac{1}{J} \sum_{j=1}^{J} V_{j}(t)$, an index of average relative capability gains can be construed as

$$
\phi(T)=\frac{1}{T} \sum_{t=t_{0}}^{T} \frac{v(t)-\underline{v}(t)}{\bar{v}(t)-\underline{v}(t)}
$$

where $T$ is the time period considered and $t_{0}$ is a sufficiently large transitional period to render initial conditions irrelevant.

The difference $v(t)-\underline{v}(t)$ is the system's average gain resulting from the actual choise over the potential maximum one $\bar{v}(t)-\underline{v}(t)$. Index $\phi(T)$ takes economically meaningful values between 0 and 1 for $T \rightarrow \infty$.

In following section we run simulations with population of firms is $J=64$., by setting the number of inward neighbours $k_{i n}=3$ and the spillover coefficient $a=.25$.

\section{Simulation Results}

The model shown in section two has been simulated by tuning two crucial variables: (i) the mean waiting time of shock arrivals, $\mu$, determining a change of $C(t)$ and (ii) the neighbour searching 
routine that we call $\tau$. The latter variable is defined in terms of the population of firms' space spanned when searching for a new neighbour. At one extreme, if a broad capacity to investigate and gather information from all firms is assumed, this space is the whole population of firms in the economy $J$; otherwise, any intermediate number up to the other extreme where firms are limited to explore only their extant neighbourhood. Thus, by calling $\pi$ the probability of exploring across the entire firm space once in a given number, $n$, of attempts to change a randomly chosen neighbour, we define $\pi=\frac{1}{n}$ and $\tau=\frac{1}{\pi}$. It follows that when $\tau=1,(\pi=1, n=1)$, the search routine is such that firms always look across the whole economy for a new neighbourhood member but $\tau$ tends to infinity, $\tau \rightarrow \infty$, when searching is entirely restricted within their neighbourhood ( $\pi \rightarrow 0$, they never look outside). Intermediate values state the number of times searching occurs inside and just once outside.

\subsection{Search routines and efficiency}

It is in the model's logic that performance be gradually improved through contributing neighbours' adjustment whatever the choice of a searching routine. This steady improvement is more or less deeply upset by negative as well as positive shocks arriving randomly according to different waiting times, $\mu$. In the following diagram we plot the last index defined in the previous section, $\phi(T)$ As it can be seen, it is indeed confirmed that for any average waiting time, a searching strategy confined to one's own neighbourhood with some across-the-board exception brings about a clear relative improvement in the system's average innovative capability but up to a certain point. The differently coloured lines correspond to as many shock mean waiting times in ascending order. 


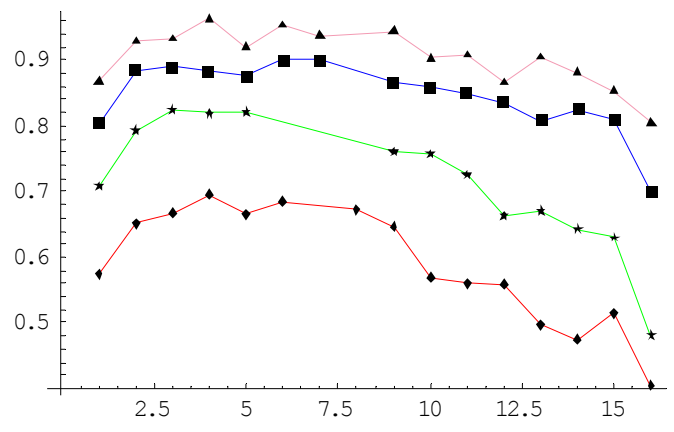

Figure 1

That performance improves as the mean waiting time, $\mu$, rises is obviously the case since firms have more time to adjust and absorb random perturbations. What is more interesting, however, is the clearly contrasting behaviour of search routines. It clearly appears that whatever $\mu$ turns out to be, performance is noticeably improved towards the theoretical maximum as firms cease to randomly scout the entire economy when they get the chance to substitute the least performing neighbour. As they concentrate on a local rather than a global search, better results are obtained at least up to a neigbourhood of $\tau^{*}$ : this means that it pays to search within one's own neighbourhood and yet to look over the entire economy approximately once every approximatively $\tau^{*}$ times. Insisting on an even more localised search, however, invariably worsens performance. The reason why this happens is quite straightforward. Localised search has both an advantage and a disadvantage over the purely random one across the whole economy. The former lies in the fact that if a firm has high performing contributors, their own contributing neighbours are also likely to be high performing ones; thus, excluding the least performing of these high contributors to substitute with one of its own neighbours carries a high probability of chancing on an even more performing contributor. On the contrary, if a firm were to carry its search for substitution over the entire firm space, it would stand an equal probability of finding either a poor or a high performing new neighbour.An improvement in a neighbour's contribution could then come from a comparatively mediocre firm The latter lies in the fact that if a firm gets locked in poor performers, localised search is likely to perpetuate mediocre performance. As a consequence, there is a trade 
off between localised and across-the-board search. It pays to search locally but to renew the pool of neighbours by searching randomly across the whole economy every now and then. To clarify this point, consider the case of a firm that starts its process by local search having high performers: it would clearly manage to quickly raise its technological capability. If, however, one of its neighbours is hit by a negative shock sizably lowering its performance, the firm would cease to obtain high positive externalities and its capability growth would consequently plunge. Exclusively localised search would then leave this firm in a poor performing environment until positive shocks arrive to lift it out of this lock in: a further drawback is that positive and negative shocks are equally probable for any given waiting time $\mu$. The only way to break the lock in is to search outside one's own neighbourhood. It follows that a good routine is one that considers local search to exploit the probability of finding high performers but that periodically searches abroad for better ones to avoid poor performing lock-in's.

\subsection{Outward neighbourhoods and paradigm setters' emergence}

The evidence shown above indicates that there is an important relationship between searching routines and their efficiency. It follows that farsighted search still has an important role to play and that short-sightedness and farsightedness must be balanced out to yield greatest search efficiency. Results provided by simulations on how technological leadership evolves help to explain this emergent phenomenon.

The following figures show diagrams in which the x-axis represents classes of inward neighbours (i.e. the number of firms by which each is observed) and the y-axis represents the average number of firms belonging to each class within the considered time span. The population of firms is made up by 64 individuals split in 32 classes: thus, the first class in each diagram includes firms having either 1 or nought neighbours, the last either 62 or 63 neighbours. We consider as paradigm setters firms lying in the last two classes, that is by almost $95 \%$ of all firms. We begin our analysis by discussing the case in which $\mu=8$ and $\tau=1$. 


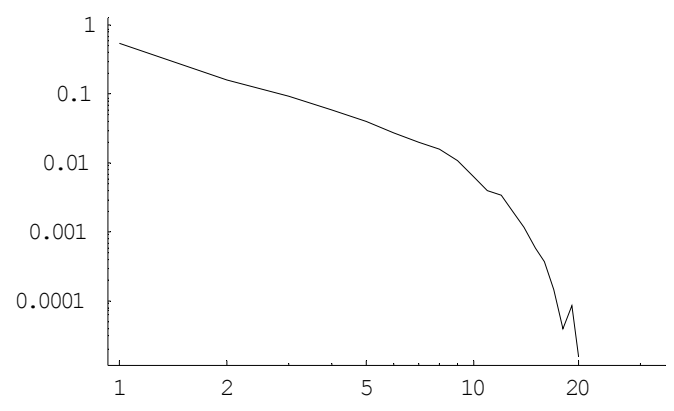

Figure 2: Time average of quantiles in the $\log -\log$ space.

Figure 2 reveal that the last classes are empty. This means that when the mean waiting time of shock arrivals is too low and the search routine randomly spans the whole economy, the probability of paradigm setters' emergence is nil Furthermore, as we have argued above, lack of adjustment time and broad randomness of searching hinder quite a few firms from seeking out high performers and, thus, neighbourhood configurations remain suboptimal. This pattern retains its main features as long as the search routine remains defined by $\tau=1$ even if mean waiting times lengthen to allow sufficient neighbourhood adjustment. Random choice of new neighbours from the entire firm space implies performance mediocrity but also avoidance of lock-in for better or for worse no matter what the shock arrival waiting time is. Firms keep on changing their neighbours so much that no particular firm is allowed to fix its technological capability as a paradigm for the rest of the economy: as a result, the last classes remain empty and no paradigm setter emerges. As routine search becomes more local paradigm setters begin to emerge and as searching approaches $\tau \rightarrow \infty$, that is when the choice of new neighbours is restricted exclusively within the narrow bounds of a firm's own neighbourhood, the same firms tend to persist as paradigm setters. The economy ends up by having a distribution featuring very few paradigm setters and a multitude of firms observed by no one else: firms with zero outward neighbours. This result occurs independently of $\tau$ provided that $\mu$ is sufficiently high, i.e. if shocks hit the economy sufficiently rarely.

The following figure illustrates this point: 


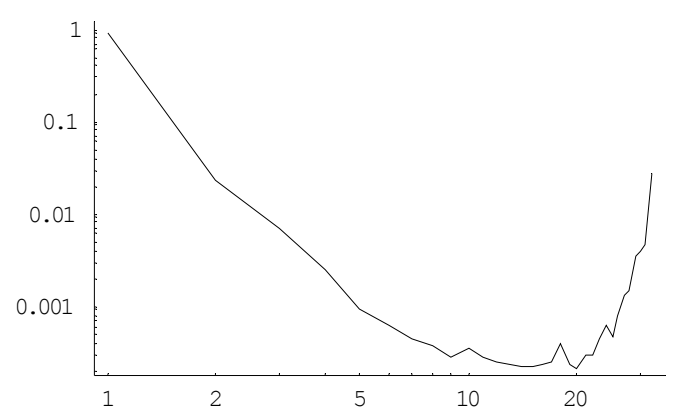

Figure 3: Time average of quantiles in the $\log -\log$ space

This diagram shows the frequency distribution for $\tau=64$ and $\mu=64$, respectively. In this case the probability that there be firms with 62 or 63 technological onlookers is significantly positive. To compound these results, it is important to consider what happens at the two or three firms that temporarily emerge as leaders and eventually become paradigm setters as evolution unfolds, shocks arrive and adjustment takes place. Simulations show that after some time about two firms rise to the role of paradigm setters as the ones that most other firms elect as their inward neighbours: they possess, in other words, the highest number of outward neighbours.

It is apparent that the emergence of paradigm setters is related to the achievement of better performance. Our data suggest that paradigm setters' emergence precedes the attainment of maximum performance, and as such it appears to be its prerequisite. Lock-in and renewal by looking over the whole economy balance out in the region around $\tau^{*}$. Nevertheless, as $\tau$ rises past the observed peak and search becomes ever more local, the lock-in effect becomes pervasive and average capability falls. There is an interesting implication in this statement: past the maximum $\tau^{*}$, while paradigm setters are the most observed firms in the economy and as such set the technological standards through their spillovers, they need not be the economy's most performing members. It is quite likely, in fact, that there may be firms with very high innovative capability that are left unchosen because never discovered by a very local search routine. In this sense, the system is likely to lock into a progressively more inefficient leadership. 


\section{Conclusions}

The foregoing analysis highlights the importance of searching and networking in fostering the development of technological capabilities to innovate in a context of bounded rationality. Firms obtain information and learn when crucially placed in a cognitive and information providing neighbourhood. Technological spillovers flow and give other firms the opportunity to learn only if networks form to give shape to searching and make learning possible. This paper depicts this process as an effort by firms, which do carry out their own in-house innovation capability building, to seek out high performers able to contribute to the latter. Routines differ according to the breadth of this search. Thanks to a simulated, simple, linear system, it is shown that broad, economy wide search routines are inefficient. The system's average innovative capability rises when searching is more local and firms are constrained to seek out new neighbours only among their discarded neighbour's neighbours. Up to a point. While the said ploy implies a technological lock-in, the probability of finding better performing firms increases. This result, however, occurs only if firms still carry out a broad, economy wide search with a frequency that simulations approximately identify. Thus, tuning short-sightedness and far-sightedness improves the system's innovative efficiency. Past a given combination of the two, the system slides towards increasing mediocrity but paradigm setters emerge as permanent and systematic feature of this hypothetical economy. The more local search is, the greater is the likelihood that paradigm setters' emergence occurs. When searching is highly and in the limit exclusively local, a lock-in into mediocre paradigm setters takes place and neighbourhoods are no longer providers of efficient spillovers.

Exogenous shocks set another time scale to the system and upset the learning and neighbour choosing adjustment. As shock arrival waiting time increases the system's technological efficiency rises but there is very little impact on paradigm setters' emergence that, therefore, appears to depend only on the chosen routine pattern. 
Further research is required to investigate the effects of a lopsided distribution of spillover coefficients $\left(a_{i j}^{\prime} s\right)$ and the possibility that inward neighbourhoods may evolve to produce emergent properties of the system's innovative capabilities.

\section{References}

Albert, R. and A.-L. Barabasi, 2002. Statistical mechanics of complex networks. Reviews of Modern Physics, 74, 47 - 97.

Arenas, A., A. Diaz-Guilera, C. J. Pérez and Fernando Vega-Redondo, 2002. Self-organized criticality in evolutionary systems with local interaction. Journal of Economic Dynamics and Control, 26, 2115-2142.

Arenas, A., A. Diaz-Guilera, X. Guardiola, M. Llas, G. Oron, Conrad J. Pérez and F. VegaRedondo, 2001. New Results in a Self-organized Model of Technological Evolution. Advances in Complex Systems, 1, 1-12.

Cowan, R. and N. Jonard, 2004. Network Structure and the difusion of knowledge. Journal of Economic Dynamics and Control, 28, 1557 - 1575.

Kirman, A., 1997. The Economy as an Evolving Network. Journal of Evolutionary Economics, 7, 339-353.

Kirman, A., 1997. The Economy as an Interactive System. In Arthur W.B., Durlauf, S.N and Lane, D. (Eds)., The Economy as a Complex Evolving System II, Santa Fe Institute, Santa Fe and Reading, MA, Addison-Wesley.

Watts, D.J. and S.H. Strogatz, 1998. Collective Dynamics of 'small world' networks. Nature, $393,440-442$. 